770 The LaNCet,] MR. W. G. SPENCER: APPENDICITIS TREATED BY IMMEDIA'TE OPERATION. [SEPT. 14, 1907.

disproof of the contentions it was supposed to uphold. He apparently gives it as a typical instance of a diphtheritic pulse. It evidently belongs to the period we are discussing. The rate and regularity of the pulse were noted at short intervals of two or three hours throughout a 27 hours' period. The details are so fully given that little doubt is left but that it is no more than a case of respiratory irregularity in spite of the history of cardiac dilatation. The variation in rate from 120 down to 66 in the 24 hours is seen to be a more or less regular movement, consisting of one large wave and depression with a small reflowing wave at the intervening 12.hourly period. The pulse is marked "regular" at the maximum acceleration and "very irregular" at the minimum rate, with varying degrees of irregularity at the stages intervening. The same writer in another place shows that he also makes no distinction between the different forms of irregularity. In referring to the history of a former case he finds the pulse was irregular and on this concludes that the heart was specifically affected. No allusion has been made to the possible effect of a diseased vagus nerve in the earlier stages of diphtheria. Whatever effect it may have in producing true irregularity-though I believe the affection of the heart wall is the main consideration at this time-it has obviously no effect upon the frequency of occurrence of the pulsus paradoxus, as is evident from the above comparisons of different diseases and from the presence of the latter in health.

In most cases respiratory irregularity of the pulse in children persists throughout their stay in hospital-that is, for periods of from four weeks to as many months-especially in marked cases, although they have otherwise regained their normal state of health. There is, however, always a great tendency for it to grow less with the return to the normal vascular tone and, as might be expected, adolescents and adults are most likely to show improvement in this respect. Owing to the limitations of space I have refrained from giving instances of actual cases, numbers of which might have been produced to illustrate every point of this paper. I might add that from the knowledge of the capricious appearance and disappearance and persistence of respiratory irregularity of the pulse and from experience gained in hunting up old patients many months after discharge from hospital-cases of typhoid fever and scarlet fever, as well as diphtheria-I have much difficulty in taking seriously many of the cases described in the literature of the subject who are stated to have lost an irregularity of the pulse which they had exhibited during many weeks and months and on the disappearance of which they had been promptly discharged. I fear that many of these cases visited afterwards in their own homes would be still found with the same peculiarity of pulse for which they had been so long detained in hospital.

In conclusion, a proper understanđing of this subject has an important bearing upon the prognosis and treatment of the disease. More than one writer has urged that the patient should be retained in bed for at least three months ${ }^{7}$ if irregularity of the pulse continues. Should irregularity, however, be as unimportant as it has been shown to be in seven-eighths of all cases, it becomes rather a serious matter, economically and medically speaking, to keep a practically healthy child in bed for a month longer than is necessary, particularly as a condition of slight general asthenia is much more likely to disappear with exercise in the open air. Of course, it is none the less necessary first carefully to decide that the irregularity is no more than respiratory. The presence of cardiac signs will often help to decide this matter. Should these, however, be lacking, the pulse otherwise good and the patient apparently returned to health, he may be at once discharged, just as hundreds of such cases are discharged yearly from the various fever hospitals, assuredly none the worse for a due return to their normal state of life.

The results of observations upon patients in hospital are published with the kind permission of Dr. Philip Boobbyer, medical officer of health and superintendent of the isolation hospitals.

Bibliography. $\mathbf{1}^{1}$ Rolleston: Practitioner, vol. ii., 1904, p. 797. 2 Hibbard : Medical and Surgical Reports of the Boston City Hospital 1898. 3 Villy : Medical Chronicle, 1899, p. 391. 4 Aschoff : Brit. Med. Jour., vol. ii., 1906. p. 1103. 5 Wenckebach : Arhythmia of the Heart p. 142, 1904. 6 Barr : Brit. Med. Jour., vol. i., 1907, p. 917. 7 Bolton : THE LANCET, Feb. 4th, 1905, p. 278. Bolton: THE LANCET, Feb. 3rd, 1906, p. 282. 9 Balfour : Diseases of the Heart and Aorta, p 266, 1898 . Med. Jour., p. 369,1907 , in an article published since this was written. Nottingham.

\section{RESULTS IN APPE'NDICITIS TREATED BY THE IMMEDIATE AND COM PLETE OPERATION.}

By WALTER G. SPENCER, M.S. LOND., F.R.C.S. ENG., SURGEON TO THE WESTMINSTER HOSPITAL.

IT is important to note that in spite of the prevailing severity of appendicitis a continuous reduction in mortality can be achieved by operating systematically as soon as a diagnosis has been made, with the determination to remove the appendix in all cases. In a series of 100 strictly consecutive cases up to Jan. 31st, 1905, which I communicated to the Royal Medical and Chirurgical Society at the discussion on appendicitis, the mortality was 15 . In a similarly consecutive series of 100 cases ending June 13th, 1907, the deaths numbered seven. Of these three died in consequence of pre-existing disease whilst recovering after operation for an intercurrent appendicitis, leaving four deaths in which the diagnosis and operation were too late. There is this special value in the comparison of the two series: the great majority of the cases in both series was first seen and approximately diagnosed by the same practitioners. The diminution in mortality is therefore to be accounted for by the earlier time at which these practitioners have found themselves able to make a diagnosis and to put the case under my care. For all the 100 cases were thus transferred to me after diagnosis except two nurses with acute perforation. This immediate operation has not entailed errors of diagnosis ; a bimanual examination in particular has determined the disease in the subacute cases, and in acute cases, when the appendix could not be felt, the signs of early septic peritonitis have been a sufficient indication for immediate operation.

The superiority of the immediate and complete operation is established not only by the series as a whole but by the cases individually. No amount of experience affords grounds for a certain prognosis, so that any delay in operating simply leaves the patient to chance. Anything short of the complete exposure and removal of the appendix fails to reach the deepest focus of suppuration. It may be taken as a rule that until the appendix has been shelled out septic pockets remain unopened. Consequently in this series there have been no lingering cases except the fatal one (6) in which all the extent of suppuration could not be reached at the operation.

A table of the 100 cases illustrates the greater severity of appendicitis in young subjects and in males:-

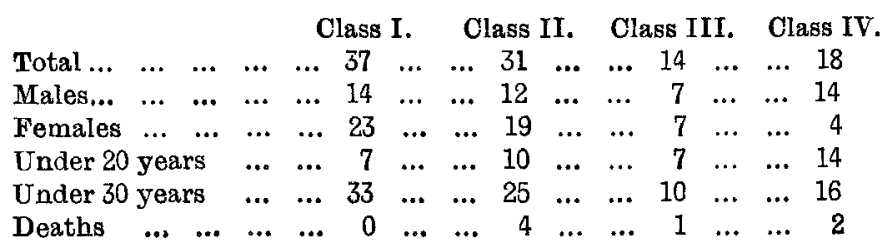

CLASS I.-Removal of the appendix and the complete suture of the abdominal wall. 37 cases; no deaths. No hernias or fistulas afterwards. These were either cases in the interval between the attacks or early acute and subacute cases in which it appeared that drainage might be dispensed with. All had disease of the appendix to some degree; no doubt some would have recovered from the particular attack without operation, others would have become rapidly worse had the operation been delayed. Beforehand one could not have distinguished them.

Class II.-Cases of acute septic peritonitis following gangrenous, perforating, and subacute appendicitis. Appendicectomy and suture of the abdominal wall except for a drain laid obliquely through the wall, removed after a day or two. The peritoneal cavity, the pelvis in particular, was sponged out; the septic fluid removed varied from 30 to 500 cubic centimetres, but the peritoneum generally appeared capable of still absorbing. 31 cases ; four deaths. All the recoveries were complete, without hernias or fistulas.

of the deaths:

(1) A male, aged 22 years, died on the fifth day after operation. The wound and lower part of the peritoneal cavity appeared to be healing well. There was an acute ulcer of the stomach with septic sub-diaphragmatic peritonitis. He was supposed to have had three previous attacks of appendicitis. The appendix at the operation was found 
kinked and subacutely inflamed, with some peritonitis around; the gastric ulceration remained unsuspected until the postmortem examination.

(2) A female, aged 27 years, died four days after operation for acute gangrenous appendicitis with peritonitis and tympanites, the acute symptoms having set in about 24 hours before operation. At the post-mortem examination the lower half of the abdomen and wound appeared to be healing satisfactorily. The stomach showed the remains of widespread gastric alceration and the upper small intestines were much dilated. She had suffered from dyspepsia for 10 years and had been accustomed to take enormous quantities of commercial bicarbonate of soda.

(3) A man, aged 35 years, was seized with abdominal pain and diarrhoea. His stools came to contain large quantities of blood; later they mainly consisted of pus. $\mathrm{He}$ rapidly wasted, suffered from aphthæ, and died in the sixth week of the illness. On the fourteenth day he had commenced to have pains in the ileo-cæcal region; on the eighteenth day he was operated upon and an acutely intlamed appendix was found bound down by old adhesion and there was local septic peritonitis. After the removal of the appendix all peritoneal symptoms ceased and the wound healed well. The diagnosis of the intestinal affection proved impossible, as a post-mortem examination was refused; it was supposed to be a case of ulcerative colitis, but there was no fibrin or mucus in the stools and the course was rapid. All the reactions of typhoid fever were negative; there were no fever and no lencocytosis except in connexion with the intercurrent appendicitis. There was no evidence of any leaking abscess.

Probably none of the foregoing three cases would have died from the appendicitis alone.

(4) A female, aged 20 years, had acute gangrenous appendicitis with peritonitis, which had commenced five days before, and there was already marked tympanites. She died about 24 hours after operation and was found to have been otherwise healthy.

Class III.-Cases of suppurating appendicitis treated by shelling out of the appendix, wiping out the affected portion of the peritoneal cavity, and afterwards filling it with gauze some cases were partly sutured. 14 cases; one death. No sinuses persisted, for the appendix was in all completely removed up to and including its funnel-like junction with the cæcum. Owing to slow healing some of the scars were not at first firm and several of the cases have been ordered belts. Possibly a case may require excision of the scar and suture in stages.

Of the death :

(5) A female, aged four years, after eight days of obscure abdominal disturbance, had marked tympanites. I found a multiloculated abscess in the pelvis around a gangrenous appendix. The child died 36 hours after operation.

CLASS IV.-Cases requiring an extensive ileo-lumbar in cision for the removal of the appendix and the complete evacuation of pus and fibrinous masses. All the suppurating carity was packed with gauze whilst pushing back the intestines. Secondary suture was done as soon as granulations lined the cavity. 18 cases; two deaths. All the scar remain sound and no belts or trusses have been required. The earliest secondary suture was four days after operation, the average being about nine days after. In one case in which a portion of the cæcum was already gangrenous, and a free fæcal fistula followed, the parts around were so soft that it was only after four months that the secondary suture could be undertaken, when a careful dissection and suture in stages resulted in sound healing. In another case a sinus persisted after the secondary suture. Six months later I explored to find that the sinus ran upwards through the psoas muscle nearly to the spine. A thorough scraping was followed by sound healing. In one case I made this deviation from the rule of always excising the appendix ; I had already completely exposed the area of suppuration, and isolated the crecum and appendix when the patient's condition appeared dangerous, so I wrapped up the cacum and appendix in iodoform gauze. Three days later I removed the appendix and sewed up except for a drain.

Of the deaths :

(6) A male, aged 20 years, was seized with an obscure abdominal attack immediately after a long bicycle ride, during which his pulse and temperature, after being at first raised, became normal and the temperature even subnormal. He was found to have an abdominal abscess extending from the pelvis upwards in front of the intestines, and pus came out from the foramen of Winslow; the tip of the appendix had sloughed off and there were two concretions loose in the pelvis. On the second day after operation a portion of the cæcum sloughed. After a month an attempt at secondary suture was made and failed. In the second month after operation he passed pus in his motions and septic bronchitis appeared. In the third month a left empyema had to be drained. He died ten weeks after operation. There was suppuration between the diaphragm and the liver and spleen, also in the lesser peritoneal cavity, and multiple abscesses in both lungs. The method of operating here failed because the suppuration had already extended upwards into the lesser peritoneal cavity. The pus throughout showed streptococci.

(7) A male, aged 19 years, had acute gangrenous appendicitis with septic peritonitis, followed by tympanites. He was brought in collapsed and covered with a cold sweat. The appendix had sloughed owing to two concretions; the pelvis was full of pus and fibrin with adhesions. He died six hours later. There was no post-mortem examination.

Thus it has been possible to diagnose and to operate on 100 cases of appendicitis, with a loss when the appendicitis was the sole disease of only four, a result which removes the various objections that have been raised to the method.

Brook-street, W.

\section{Clinital 望otes:}

\section{MEDICAL, SURGICAL, OBSTETRICAL, AND} THERAPEUTICAL.

\section{ACUTE ARTHRITIS COMPLICATING A CASE OF OPH THALMIA NEONATORUM.}

BY J. H. F. NUNN, M.R.C.S. ENG., L.R.C.P. LoND.

Mrs. - a healthy primipara, gave birth to a strong, well-nourished child. On the third or fourth day after birth an acute attack of purulent ophthalmia of the infant's left eye developed. The conjunctivitis was treated with boric and perchloride of mercury lotions and occasional applications of silver nitrate paint (10 grains to one ounce) as the discharge was profuse. Care was taken to allow the child time for sleep. About 24 hours after the conjunctivitis of the infant declared itself the mother developed an attack of pelvic inflammation. I obtained a distinct history of gonorrboea from the father of the child, who had been treated for the complaint, and I ascertained from the mother that she had had a discharge for some weeks previous to her confinement. After four or five days, when I hoped that the infant's right eye had been preserved, it became similarly affected to the left which was now almost well. At this juncture the metatarso-phalangeal joint of the left great toe of the baby became hot, swollen, red, and painful to touch, the condition being similar to a comparatively mild attack of gout. The nurse was careful and from her I could obtain no evidence of a fall, bruise, burn, or any injury likely to produce the condition. The joint became slightly better, but on the left eye becoming reinfected (this time the condition was worse than before, so that a large central opacity developed which eventually nearly dis. appeared) the joint again became more acutely inflamed. The arthritis gradually subsided without leaving any stiffness or trace of the inflammation. 'The child's digestion and bowels were attended to and the joint was occasionally fomented and protected with cotton wool, beyond which no treatment was attempted. The reduction of the discharge from the eye by the use of silver nitrate appeared to coincide with an improvement in the joint, and when the paint was not ased for 36 hours there was not only an increase in discharge from the eye but also in the swelling of the joint.

Judging from the usual course of gonorrhcal arthritis, and from the fact that the child was healthy and its strength was being maintained, and that the apparent cause was being treated and thereby diminishe $d$, I considered the r $r$ ignosis to be favourable.

I mention the case because the complication was unexpected, and an ophthalmic surgeon of experience could not recall a similar occurrence; neither had I during 15 